\section{MS47-O4 Nikola Tesla, scientist and inventor - discovery of X-rays}

\author{
Stanko Popović1 ${ }^{1,2}$
}

1. Croatian Academy of Sciences and Arts, Zagreb, Croatia

2. Department of Physics, Faculty of Science, University of Zagreb, Croatia

email: spopovic@phy.hr

Miraculous inventions and scientific achievements have made Nikola Tesla, the Croatian inventor (The New Yorker 2013), world-famous. There are hundreds of books and papers dealing with the life and work of Tesla that confirm the above statement. In 1960 the term tesla, $\mathrm{T}$, was given to the SI unit of the magnetic field. Starting in 1894 Tesla experimented with mysterious shadowgraphs similar to those that later were studied by W. C. Röntgen (L. I. Anderson, 21st Century Books, 1994). Tesla was aware of an unknown very special radiation that had damaged film in his laboratory, later identified as Röntgen rays or X-rays. Unfortunately, much of his early research was lost when his laboratory in New York was burnt down on March 13, 1895. Röntgen published his discovery on November 8,1895 . In the beginning of 1896 Tesla proceeded with experiments in $\mathrm{X}$-ray imaging, designing a high energy unipolar vacuum tube that had no target electrode. The electrons were accelerated by peaks of the electric field produced by the high-voltage Tesla coil. Tesla realized that the source of $\mathrm{X}$-rays was the site of the first impact of electrons within the tube. Tesla devised several experimental set-ups to produce X-rays, that were of much greater power than obtainable with ordinary apparatus. He stated that the cathodic stream was composed of small particles, and that the produced X-rays were transverse waves possessing many properties of light. Tesla described his experiments in a series of papers in Electrical Review New York, the first paper appearing in March 11, 1896. Tesla gave Röntgen full credit for his discovery. Röntgen congratulated Tesla on his sophisticated images, wondering how he had achieved such impressive results. Tesla commented on physiological hazards in working with X-rays and gave recommendations for protection. Therefore, there is much evidence that confirm the legacy of Tesla in the discovery of X-rays. His lecture before the New York Academy of Sciences in 1897 validated to some degree his primacy in research of X-rays. One will never know who would have won the Nobel prize for the discovery of X-rays if Tesla's work had not been lost by fire. The least one can do is to appreciate his pioneer work in this field (Hrabak, M. et al. (2008). RadioGraphics 28, 1189).

Keywords: discovery of X-rays, Nikola Tesla

\section{MS47-O5 European crystallography before the discovery of X-rays}

Jean-Louis Hodeau ${ }^{1}$, Rene Guinebretiere ${ }^{2}$

1. Univ. Grenoble Alpes, Institut Neel - CNRS, 38042 Grenoble, France

2. ENSCI, SPCTS, 87068 Limoges, France

email: hodeau@neel.cnrs.fr

The origins of crystallography started with humanity's interrogation with crystals. From the end of the 16th century, scientists found that minerals like quartz regularly display angular shapes that are unlikely to be random. De Boodt, Kepler, Hooke, Huygens and Steno considered the cause to be related to the internal nature of the crystal. In the 17th century Steno first suggested that the hexagonal form of quartz crystals was preserved during growth. The term "Crystallography" was first used in 1723 by Cappeller.

Crystallographers in the 18th century managed to form a picture of crystal's internal structure on the basis of their external geometry. Romé de l'Isle was inspired by Linnaeus's biological classification system and proposed using the shape of the crystal as a mean of classification. From his observations of scraps of broken calcite, Haüy constructed models of crystals by stacking small bricks together, bricks which he called "molécules intégrantes". All these investigations gave rise to this new science of "crystallography", one of the oldest of the "physical" sciences, together with astronomy, mechanics and optics.

In the 19th century, German and French researchers introduced the concepts of lattice, axis, centre, and mirror plane of symmetry as criteria to classify crystals. Weiss rejected Haüy's "molécules intégrantes" theory and led a German school advocating the use of symmetry in crystals. Hessel and Frankenheim showed that there are only 32 ways of combining these symmetries. In 1840, Delafosse replaced Haüy's solid little bricks with the notion of volumes formed of space and molecules. Bravais described the sum of the volumes as a lattice system repeating itself, with an identical motif at each lattice point, and showed that there are only 14 types of lattice system. At the end of the 19th century, the hypothetical molecules were replaced by more complex wallpaper-like patterns containing atoms and new symmetries. These symmetry patterns were hypothesised by Sohncke and then recorded by Schoenflies and Fedorov. This classification of crystals based on their symmetry and lattice structure is still with us today. It is invaluable for studying the physical properties of crystals (optical, mechanical and thermal).

We can see that in the early 20th century, before the first X-ray diffraction experiments allowed to "see inside" crystals, crystallographers had already established a body of experimental and theoretical knowledge of them. 